\title{
A robust WENO scheme for nonlinear waves in a moving reference frame
}

\author{
Kontos, Stavros; Bingham, Harry B.; Lindberg, Ole; Engsig-Karup, Allan Peter
}

Published in:

Journal of Hydrodynamics

Link to article, DOI:

10.1016/S1001-6058(16)60652-2

Publication date:

2016

Document Version

Peer reviewed version

Link back to DTU Orbit

Citation (APA):

Kontos, S., Bingham, H. B., Lindberg, O., \& Engsig-Karup, A. P. (2016). A robust WENO scheme for nonlinear waves in a moving reference frame. Journal of Hydrodynamics, 28(3), 482-488. https://doi.org/10.1016/S1001$6058(16) 60652-2$

\section{General rights}

Copyright and moral rights for the publications made accessible in the public portal are retained by the authors and/or other copyright owners and it is a condition of accessing publications that users recognise and abide by the legal requirements associated with these rights.

- Users may download and print one copy of any publication from the public portal for the purpose of private study or research.

- You may not further distribute the material or use it for any profit-making activity or commercial gain

- You may freely distribute the URL identifying the publication in the public portal

If you believe that this document breaches copyright please contact us providing details, and we will remove access to the work immediately and investigate your claim. 


\title{
A robust WENO scheme for nonlinear waves in a moving reference frame
}

\author{
Stavros Kontos ${ }^{1 *}$, Harry B. Bingham ${ }^{1}$, Ole Lindberg ${ }^{1}$ and Allan P. Engsig-Karup ${ }^{2}$ \\ ${ }^{1}$ Department of Mechanical Engineering, Technical University of Denmark, Denmark \\ ${ }^{2}$ Department of Applied Mathematics \& Computer Science, Technical University of Denmark, Denmark \\ *E-mail: stakon@mek.dtu.dk
}

\begin{abstract}
For robust nonlinear wave simulation in a moving reference frame, we recast the free surface problem in Hamilton-Jacobi form and propose a WENO scheme to automatically handle the upwinding of the convective term. A new automatic procedure for deriving the linear WENO weights based on a Taylor series expansion is introduced. A simplified smoothness indicator is proposed and is shown to perform well. The scheme is combined with high-order explicit Runge-Kutta time integration and a dissipative Lax-Friedrichstype flux to solve for nonlinear wave propagation in a moving frame of reference. The WENO scheme is robust and less dissipative than the equivalent order upwind-biased finite difference scheme for all ratios of frame of reference to wave propagation speed tested. This provides the basis for solving general nonlinear wave-structure interaction problems at forward speed.
\end{abstract}

KEY WORDS: Nonlinear waves; WENO; finite difference.

\section{INTRODUCTION}

Exploiting the advantages of the high-order finite difference method in solving nonlinear potential flow, wave structure interaction problems for ships travelling with steady forward speed, requires robust numerical methods. The proposed method extends previous work by Engsig-Karup et al. [1,2], Lindberg et al. [3] and Afshar et al. [4] on developing robust strategies for efficient modeling of nonlinear waves and linear and nonlinear wave-structure interaction. When the linearised problem is considered, a one-point upwind-biased approximation of the convective derivatives in the free surface boundary conditions is sufficient to ensure stability (Bingham et al. [5]). For nonlinear problems however, this is not found to be robust for all combinations of ship speed and wave celerity. In this paper, a simplified version of the WENO (Weighted Essentially Non-Oscillatory) (Liu et al. [6]) finite difference scheme is proposed for stabilising the solution of the fully nonlinear problem. This scheme is tested using the nonlinear wave propagation problem in a moving frame of reference which mimics a forward speed seakeeping problem. The results of these strenuous numerical experiments indicate that it is robust and accurate.

\section{A SIMPLIFIED WENO FINITE DIFFERENCE SCHEME FOR THE CONVECTIVE TERMS}

The scheme is presented in one dimension, but for 3D problems it is applied in the same way to both of the horizontal convective terms. The WENO scheme creates two approximations of the convective derivative $\phi_{x, i}=$ $\left.\frac{\partial \phi}{\partial x}\right|_{x=x_{i}}$ using a left and a right biased stencil as shown in Figure 1. 

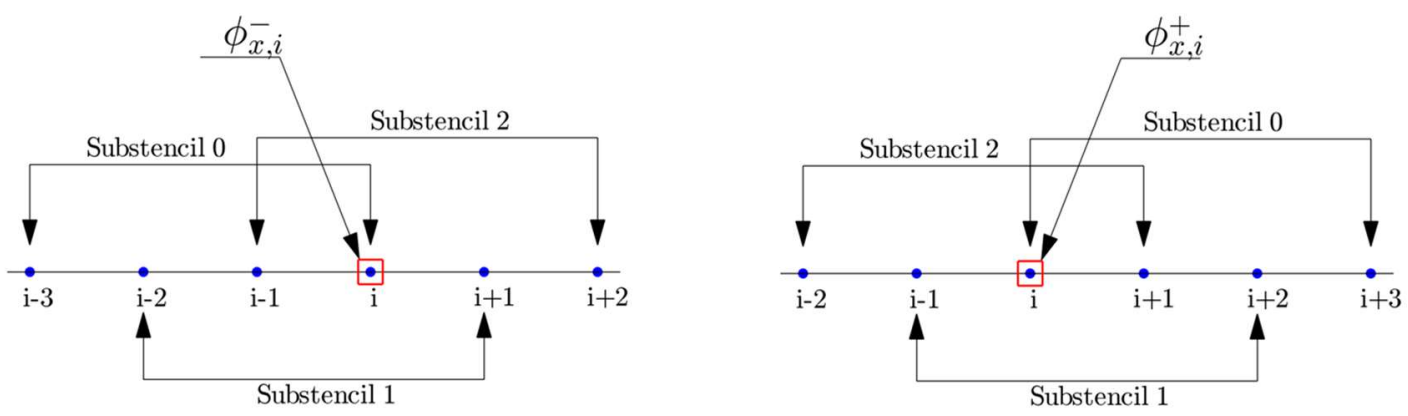

Figure 1: WENO 3 Left and Right Biased Stencils.

These two approximations are combined with an appropriate flux to give the final result. Each of these stencils consists of $r$ sub-stencils. On a left biased stencil, WENO $3(r=3)$ computes three third order approximations $\phi_{x, i}^{-, s}$ to $\phi_{x, i}$ based on each of the $s=0, \ldots, r-1$ sub-stencils. These approximations are combined using non-linear weights $\omega_{s}$ to compute the final result:

$\phi_{x, i}^{-}=\sum_{s=0}^{r-1} \omega_{s} \phi_{x, i}^{-, s}$

The $\omega$ should ensure that when the solution is locally smooth, an order $(2 r-1)$ accurate approximation is obtained. If the solution is discontinuous within the range of support of one or more of the stencils, the contributions from those stencils are weighted to zero and the accuracy of the final scheme reduces to $r$.

Following Yamaleev and Carpenter [7] the nonlinear weights are defined as:

$\omega_{s}=\frac{a_{s}}{\sum_{s=0}^{r-1} a_{s}}, s=0, \ldots, r-1, a_{s}=d_{s}\left(1+\frac{\tau}{\epsilon+\beta_{s}}\right)$

The $d_{s}$ here are constant linear weights which, in the case of a smooth solution, will provide the order $(2 \mathrm{r}-1)$ accurate result. The weights must sum to one, $\sum_{s=0}^{r-1} d_{s}=1$.

Tabulated values of $d_{s}$ for several specific cases can be found in the literature by Jiang and Shu [8], Balsara and Shu [9]. We present here a simple and general derivation procedure, applicable to any order of accuracy and easily implemented in a computer program. We seek $r$ coefficients which sum to one and set to zero the first $r-1$ truncation error terms in the Taylor series expansion of the combined derivative approximation. Thus for $r=3$, we have the system of equations

$\left[\begin{array}{ccc}S_{0,1} & S_{1,1} & S_{2,1} \\ S_{0,2} & S_{1,2} & S_{2,2} \\ 1 & 1 & 1\end{array}\right]\left[\begin{array}{l}d_{0} \\ d_{1} \\ d_{2}\end{array}\right]=\left[\begin{array}{l}0 \\ 0 \\ 1\end{array}\right]$

where $S_{s, j}, s=0, \ldots, r-1$ and $j=1, \ldots, r-1$ represents truncation error coefficient $j$ on sub-stencil $s$. For example, the derivative approximation on left substencil 0 of order $r=3$ is given by:

$\frac{1}{6 \Delta x}\left(-2 \phi_{i-3}+9 \phi_{i-2}-18 \phi_{i-1}+11 \phi_{i}\right)=\phi_{i}^{(1)}-\frac{1}{4} \Delta x^{3} \phi_{i}^{(4)}+\frac{1}{3} \Delta x^{4} \phi_{i}^{(5)}+\cdots$

where $\phi_{i}^{(n)}$ indicates the exact $n$th derivative of $\phi$ at grid point $i$. Thus, $S_{0,1}=-1 / 4$ and $S_{0,2}=1 / 3$. The set up and solution of this system can be easily implemented in a short computer program to provide the $d_{s}$ for any choice of $r$.

The $\beta_{S}$ in Eq. (2) are "smoothness indicators" which measure the smoothness of the solution on each stencil. Here again, sets of tabulated weights for specific cases can be found in Jiang and Shu [8], Balsara and Shu [9] based on rather complicated derivation procedures. We propose here a simple smoothness indicator that can be easily calculated numerically at arbitrary order: 
$\beta_{s}=\sum_{l=2}^{r}\left(\phi_{s, \mathrm{i}}^{(l)} \Delta x^{l-1}\right)^{2}$

This is a sum of all possible higher derivatives on the stencil, scaled to have units of velocity $(\mathrm{m} / \mathrm{sec})$, and is thus a measure of the smoothness of the velocity. It becomes large whenever a discontinuity exists in the solution and thus forces the associated weight to become small for that stencil. The factor $\tau$ in Eq. (2) is defined as the squared normalized highest order derivative on the full stencil of $2 r$ points,

$\tau=\sum_{i=1}^{2 \mathrm{r}}\left(\phi_{i}^{(2 r-1)} \Delta x^{2 \mathrm{r}-1}\right)^{2}$

The term $\epsilon$ in Eq. (2) is included to avoid dividing by zero and is set to $10^{-6}$.

This expression for the nonlinear WENO weights (Eq.(2)) is chosen instead of the classical weights by Liu et al. [6] because it is found to introduce less numerical dissipation into the nonlinear wave solution. For clarification, the $a$ coefficients can be expressed as in Borges et al. [10]:

$a_{s}=d_{s}\left(1+\frac{\tau}{\epsilon+\beta_{s}}\right)=\frac{d_{s}}{\beta_{s}^{z}}$

where

$\beta_{s}^{Z}=\left(\frac{\beta_{s}+\epsilon}{\beta_{s}+\tau+\epsilon}\right)$

The $\beta_{s}^{z}$ are the normalized smoothness indicators $\beta_{s}$ including the higher order information contained in $\tau$. They are all approximately equal in smooth parts of the solution. The $\beta_{s}^{Z}$ coefficient of a stencil that contains a discontinuity will be larger than that of stencils where the solution is smooth. If we consider the case where one of the stencils contains a discontinuity (e.g. stencil $s=2$ ) while the solution in stencils $s=0,1$ is smooth, it can be seen that the ratios between $\beta_{0}^{Z}, \beta_{1}^{Z}$ and $\beta_{2}^{Z}$ are larger than when using the classical weights:

$\frac{\beta_{s}^{Z}}{\beta_{2}^{Z}}=\frac{\beta_{s}}{\beta_{2}} \frac{\beta_{2}+\tau}{\beta_{s}+\tau} \geq \frac{\beta_{S}}{\beta_{2}}, \quad \beta_{2}>\beta_{S}, \quad s=0,1$

Therefore, the relative importance of stencil $s=2$ is larger and the scheme is less dissipative. More detailed analysis can be found in Borges et al. [10]. We note that as the linear weights are derived on a uniform grid, nonuniform spacing is introduced via a transformation between the physical grid and a unit-spaced reference grid.

\section{Testing the Performance of the New Smoothness Indicator}

The convergence of the simplified smoothness indicator is compared with the tabulated values from the literature for evaluating the derivative of a smooth and a discontinuous function. The smooth test function is given by:

$\phi(x)=\sin (k x),-1 \leq x<1$

And the discontinuous test function is:

$\phi(x)=\left\{\begin{array}{c}-\frac{\cos (\pi(x-0.4))}{\pi},-2 \leq x<0 \\ \frac{\cos (\pi(x-0.4))}{\pi}, 0 \leq x \leq 2\end{array}\right.$

where the offset is introduced to avoid any fortuitous cancellation of errors. No flux is used to combine the left and right biased approximations. On the left of the discontinuity the left biased stencil is used and on the right of the discontinuity the right biased stencil is used, which simulates the use of an exact Riemann solver. In the case of the smooth function there is no difference in the choice of right or left stencil. The relative error in each case is defined by: 
$\frac{\left\|\phi_{i}^{(1)}-\phi_{x, i}{ }^{ \pm}\right\|_{\infty}}{\left\|\phi_{i}^{(1)}\right\|_{\infty}}$

The convergence of the two approximations for the smooth function is shown in Figures 1-4 and we can see that the two smoothness indicators give nearly identical results and converge at the expected order of accuracy in all cases. We note that the accumulation of round-off errors prevents the relative error from dropping below approximately $10^{-13}$ as can be seen by the levelling of the high-order errors on refined grids. The results for the discontinuous function are shown in Figures 5-8 and again the two methods give nearly identical results, providing the expected rate of convergence in all cases. These results support the conclusion that the proposed smoothness indicator is able to function as well as the tabulated values in controlling the WENO scheme.
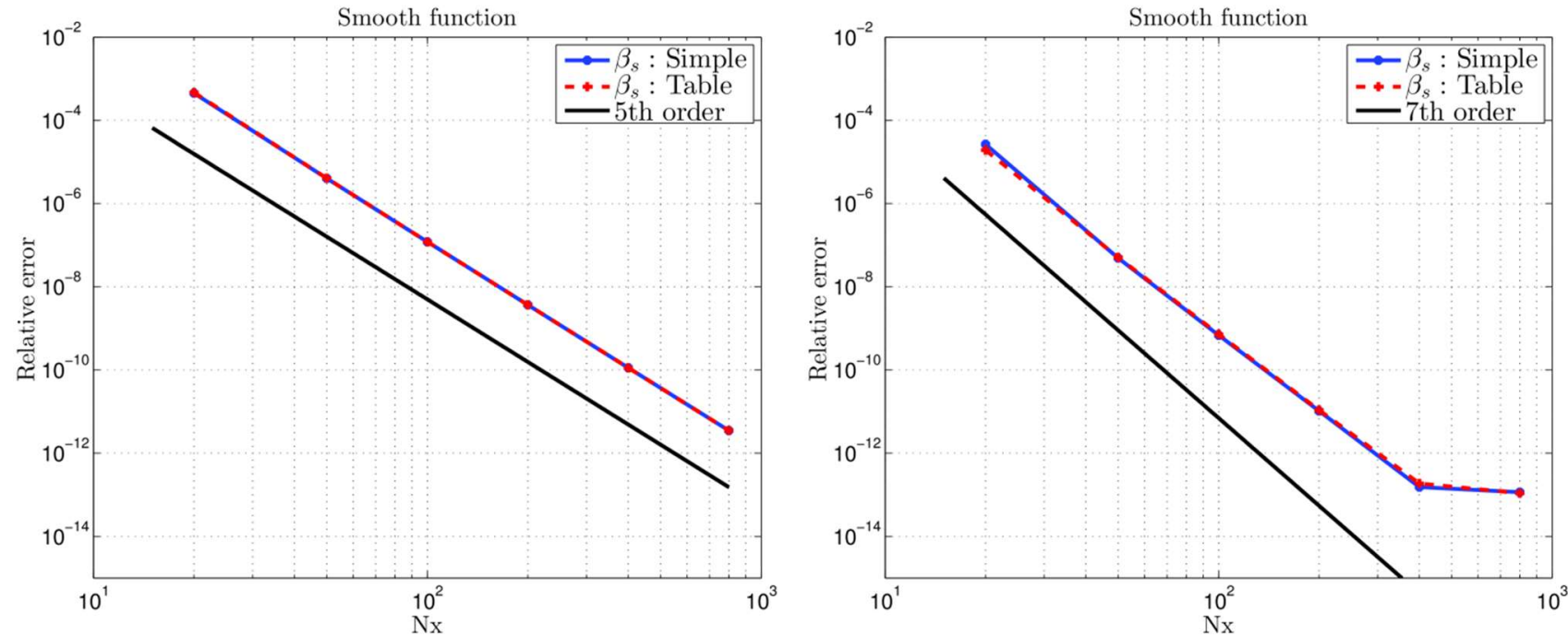

Figure 1: WENO 3 convergence.

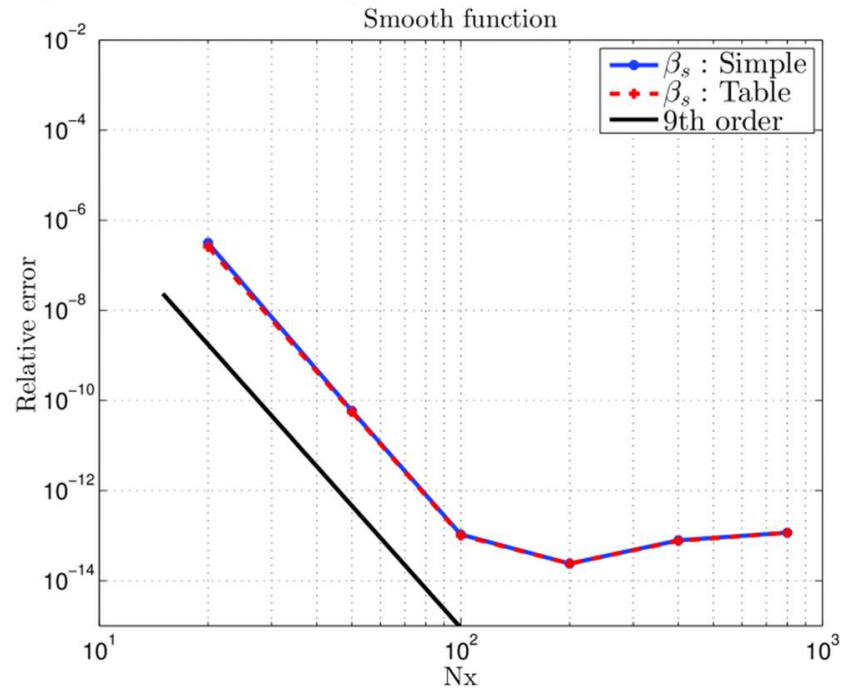

Figure 2: WENO 4 convergence.

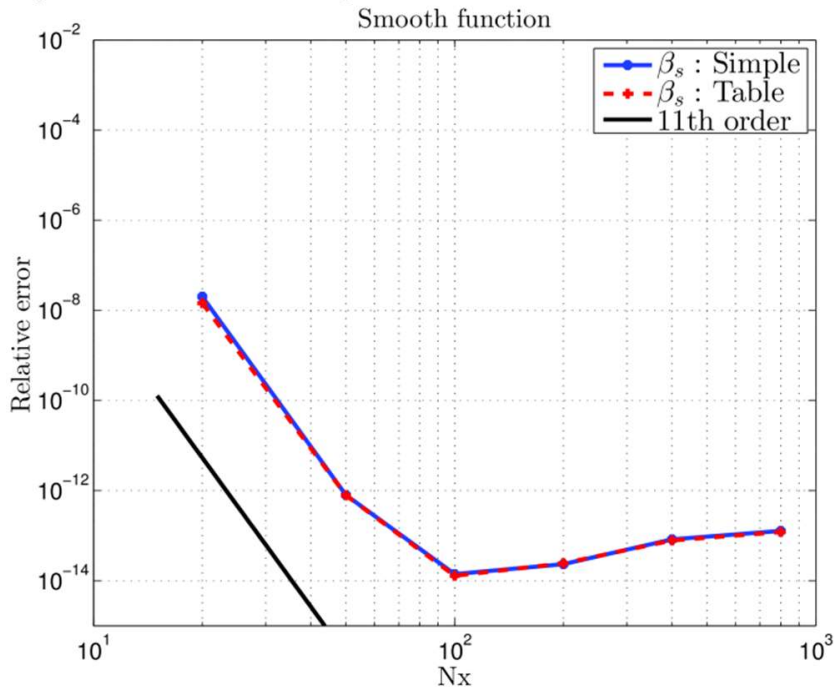

Figure 3: WENO 5 convergence.

Figure 4: WENO 6 convergence. 

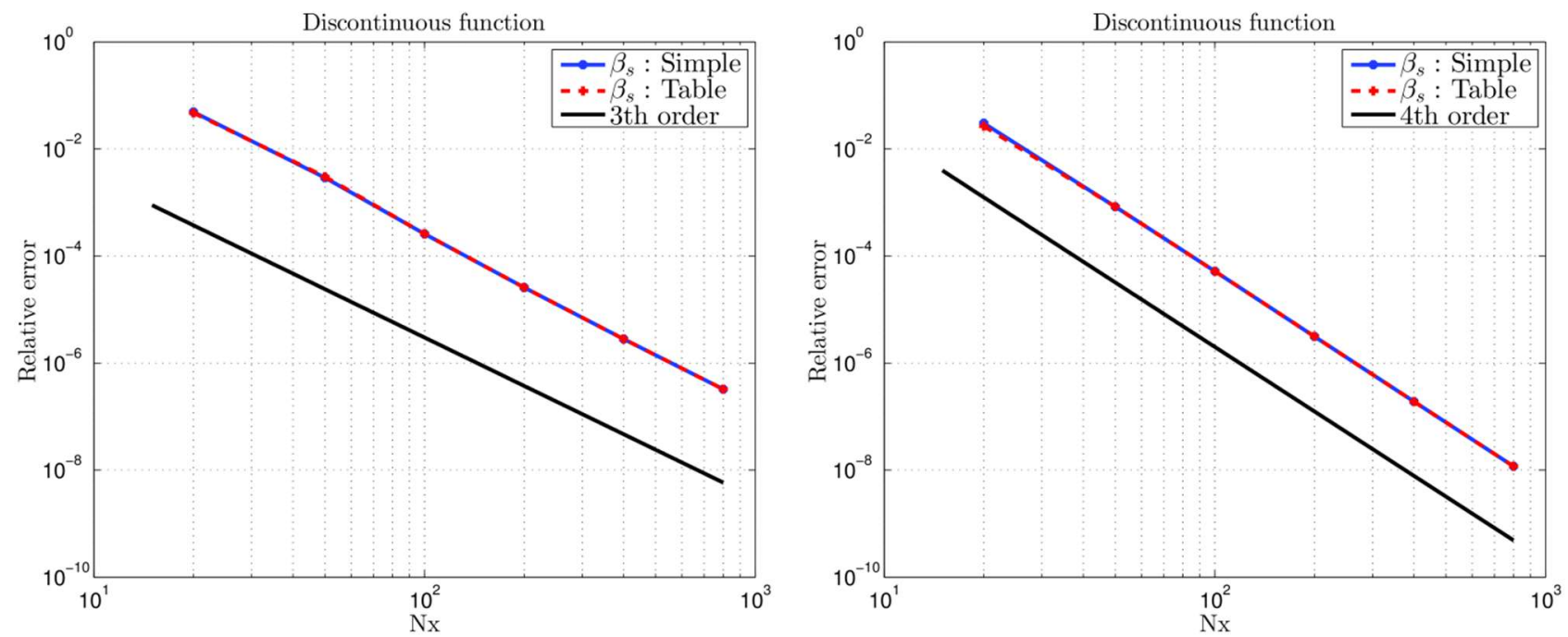

Figure 5: WENO 3 convergence.

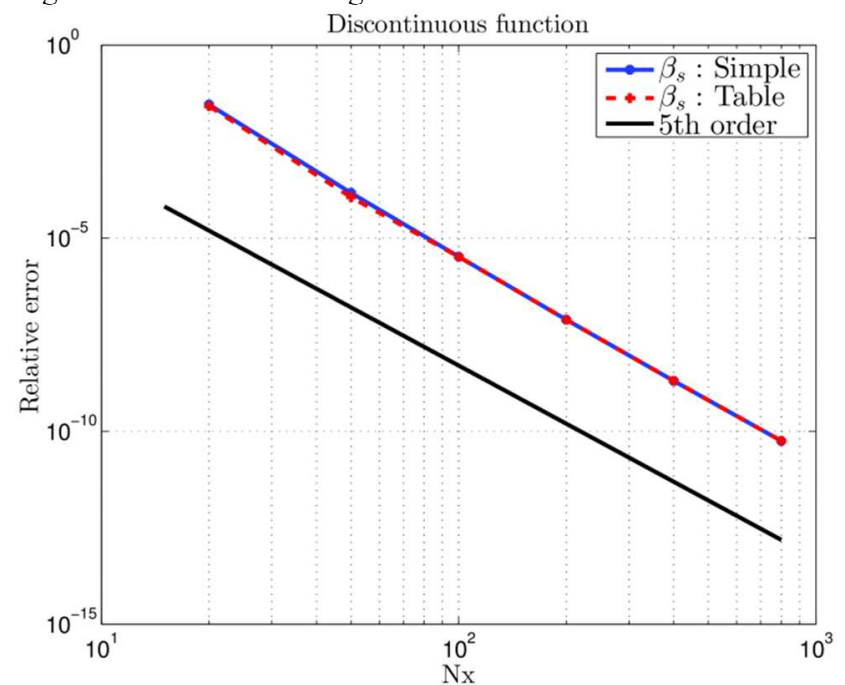

Figure 6: WENO 4 convergence.

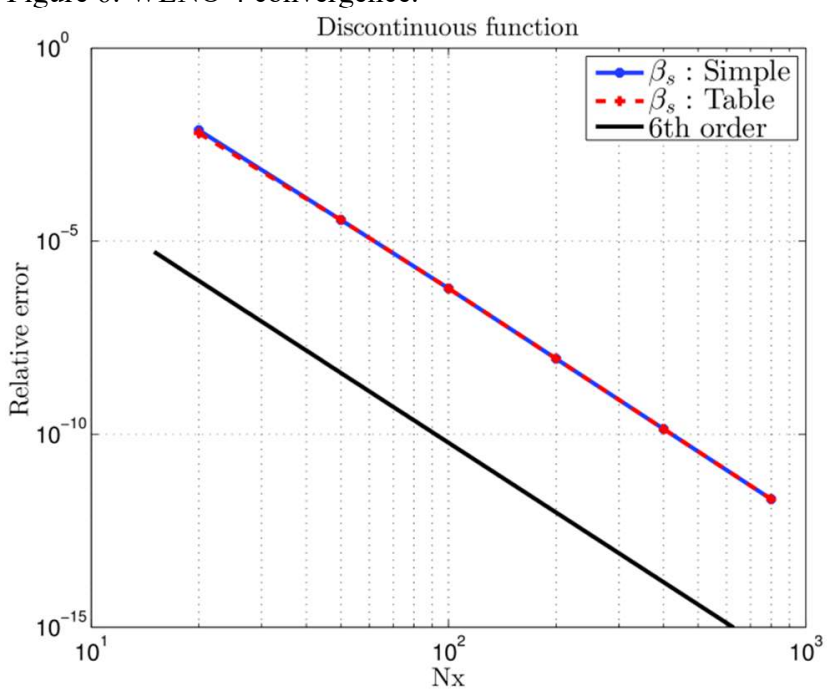

Figure 8: WENO 6 convergence.

Figure 7: WENO 5 convergence.

\section{APPLICATION TO THE FORWARD SPEED PROBLEM}

The 2D, non-linear, potential flow gravity wave problem written in a moving coordinate system with constant velocity $U$ in the $x$-direction, is expressed by the kinematic and the dynamic free-surface boundary conditions which can be written:

$\partial_{t} \eta+\partial_{x} \eta\left(\partial_{x} \tilde{\phi}-\partial_{z} \tilde{\phi} \partial_{x} \eta-U\right)=\partial_{z} \tilde{\phi}$

$\partial_{t} \tilde{\phi}+\partial_{x} \tilde{\phi}\left(\frac{1}{2} \partial_{x} \tilde{\phi}-U\right)-\frac{1}{2}\left(\partial_{z} \tilde{\phi}\right)^{2}\left(1+\partial_{x} \eta \partial_{x} \eta\right)=-g \eta$

where $\eta(x, t)$ is the free surface elevation and $\tilde{\phi}(x, t)=\phi(x, \eta, t)$ is the velocity potential evaluated on the free surface. The fluid domain is bounded by the sea bottom at $z=-h(x)$. The solution procedure of the Laplace problem is described by Bingham \& Zhang [11]. The explicit Runge-Kutta 44 method is used for time-stepping.

To apply WENO on the free surface boundary conditions, they have to be expressed in Hamilton-Jacobi form (Osher and Fedkiw, [12]):

$\phi_{t}+H(\nabla \phi)=0$.

For the spatial discretization the Lax-Friedrichs scheme is used (Crandall and Lions, [13]): 
$\widehat{H}=H\left(\frac{\phi_{x}^{-1}+\phi_{x}^{+1}}{2}, \frac{\phi_{y}^{-1}+\phi_{y}^{+1}}{2}\right)-a^{x}\left(\frac{\phi_{x}^{+1}-\phi_{x}^{-1}}{2}\right)-a^{y}\left(\frac{\phi_{y}^{+1}-\phi_{y}^{-1}}{2}\right)$

where $a^{x}$ and $a^{y}$ are dissipation coefficients for controlling the amount of numerical viscosity. They are defined as

$a^{x}=\max \left|H_{1}\left(\phi_{x}, \phi_{y}\right)\right|$

and

$a^{y}=\max \left|H_{2}\left(\phi_{x}, \phi_{y}\right)\right|$.

$H_{1}$ and $H_{2}$ here are the partial derivatives of $H$ with respect to $\phi_{x}$ and $\phi_{y}$, respectively.

The free surface boundary conditions can be expressed in the WENO formulation as:

$\partial_{t} \eta+H_{\eta}=\partial_{z} \tilde{\phi}$

$\partial_{t} \tilde{\phi}+H_{\phi}=-g \eta$

where

$H_{\eta}=\partial_{x} \eta\left(\partial_{x} \tilde{\phi}-\partial_{z} \tilde{\phi} \partial_{x} \eta-U\right)$,

$H_{\phi}=\partial_{x} \tilde{\phi}\left(\frac{1}{2} \partial_{x} \tilde{\phi}-U\right)-\frac{1}{2}\left(\partial_{z} \tilde{\phi}\right)^{2}\left(1+\partial_{x} \eta \partial_{x} \eta\right)$.

The right hand side terms, namely $\partial_{z} \tilde{\phi}$ and $-g \eta$, are considered as source terms.

The dissipation coefficients are given by

$a_{\eta}^{x}=\max \left|H_{1, \eta}\left(\eta_{x}, \widetilde{\phi_{x}}\right)\right|=\max \left|\widetilde{\phi_{x}}-2 \widetilde{\phi_{z}} \eta_{x}-U\right|$

and

$a_{\phi}^{x}=\max \left|H_{1, \phi}\left(\eta_{x}, \widetilde{\phi_{x}}\right)\right|=\max \left|\widetilde{\phi_{x}}-U\right|$.

\section{REPRESENTATIVE TEST CASES}

In a general seakeeping problem, waves will travel in all directions at a range of different speeds relative to the ship speed, and the numerical scheme must be able to handle all the possible ship speed to wave celerity ratios. Thus, we test the scheme using a set of deep water waves with $k h=2 \pi$, over a range of different ship speed to wave propagation speed ratios. The wave height is $90 \%$ of the stable limit, $\mathrm{H} / \mathrm{L}=0.1273$. The different test cases are visualized in Figures 9-14. The wave is propagated for ten periods with a fixed Courant number of $\mathrm{Cr}=0.5$ and energy conservation is monitored. The WENO 6 scheme is compared with both centered and upwinded 6th order finite difference schemes. When $U \leqslant 0$, upwinding is achieved by shifting the stencil one point to the left. For $U>0$ the stencil is shifted one point to the right, where $U$ is the ship speed. The energy conservation is monitored for each simulation and the results are presented in Figures 15-20. The centered scheme is always unstable for convective problems (and therefore not plotted for clarity). The upwind-biased scheme can handle most cases, but will always become effectively down-winded, and hence unstable, for waves which travel faster than the frame of reference and in the same direction as the frame of reference. In contrast, the WENO scheme is stable in all the tested cases. The dissipation introduced by the WENO scheme is generally as low or lower than the upwindbiased scheme, though for high-accuracy, very high-order schemes may be desirable. It should be noted that the WENO scheme was also tested with the non-linear weights of Liu et al. [6] and Borges et al. [10] but both were found to be significantly more dissipative than the ones of Yamaleev and Carpenter [7]. This provides the basis for solving general nonlinear wave-structure interaction problems at forward speed. 

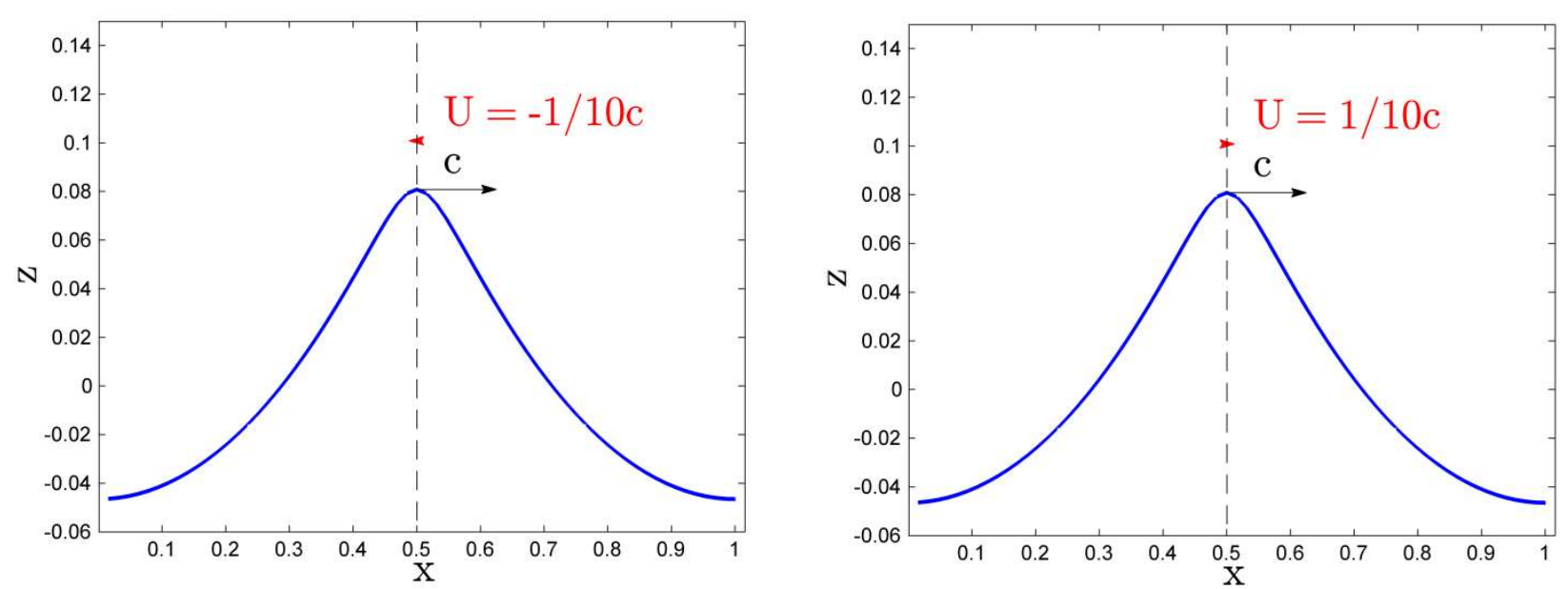

Figure 9: $U=-1 / 10 c$.

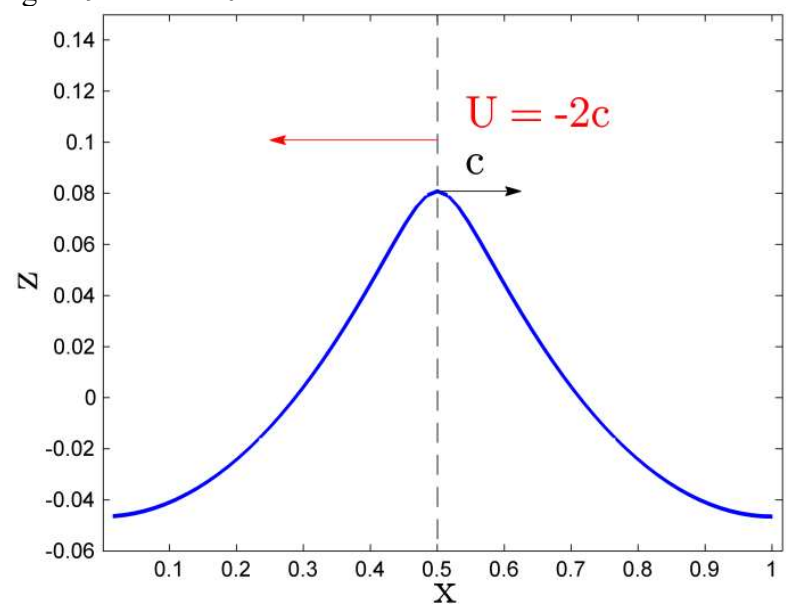

Figure 10: $U=1 / 10$ c.

Figure 11: $U=-2 c$.

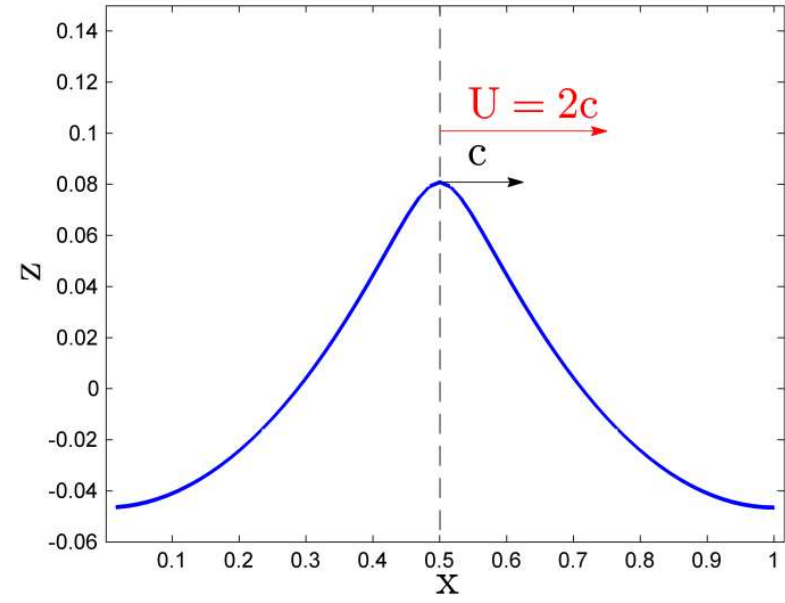

Figure 12: $U=2 \mathrm{c}$.

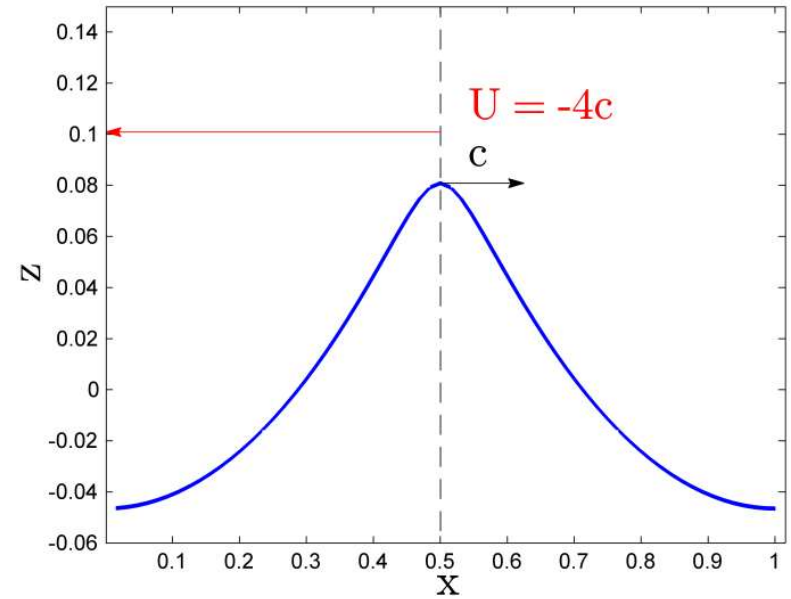

Figure 13: $U=-4 c$.

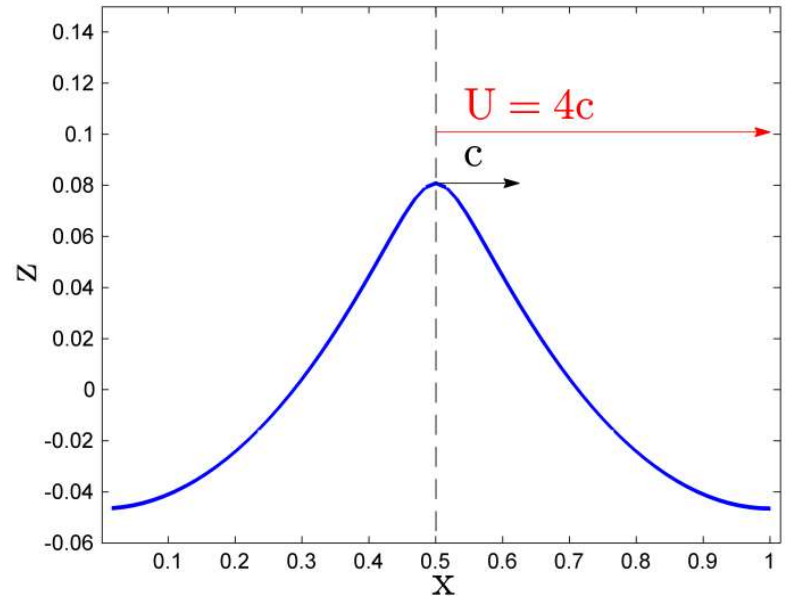

Figure 14: $U=4 \mathrm{c}$. 

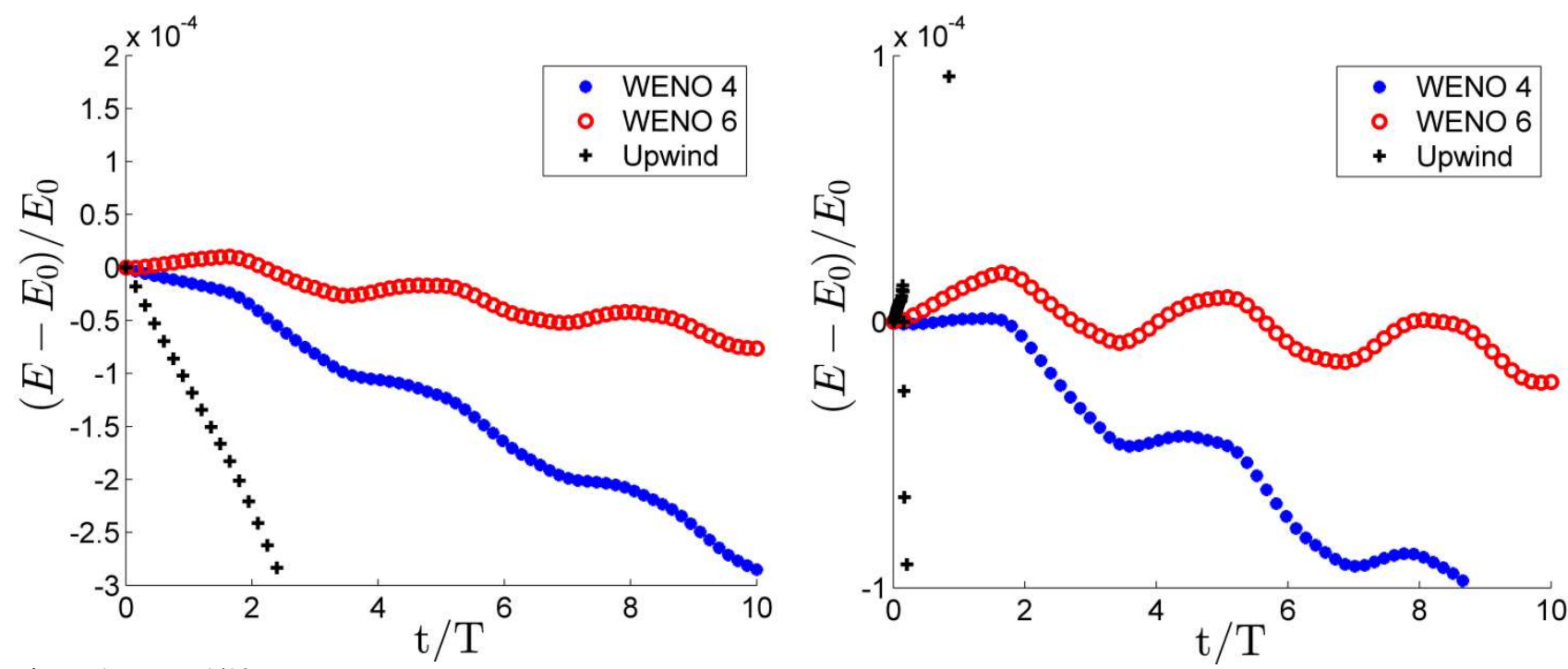

Figure 15: $U=-1 / 10 c$.

Figure $16: \mathrm{U}=1 / 10 \mathrm{c}$.
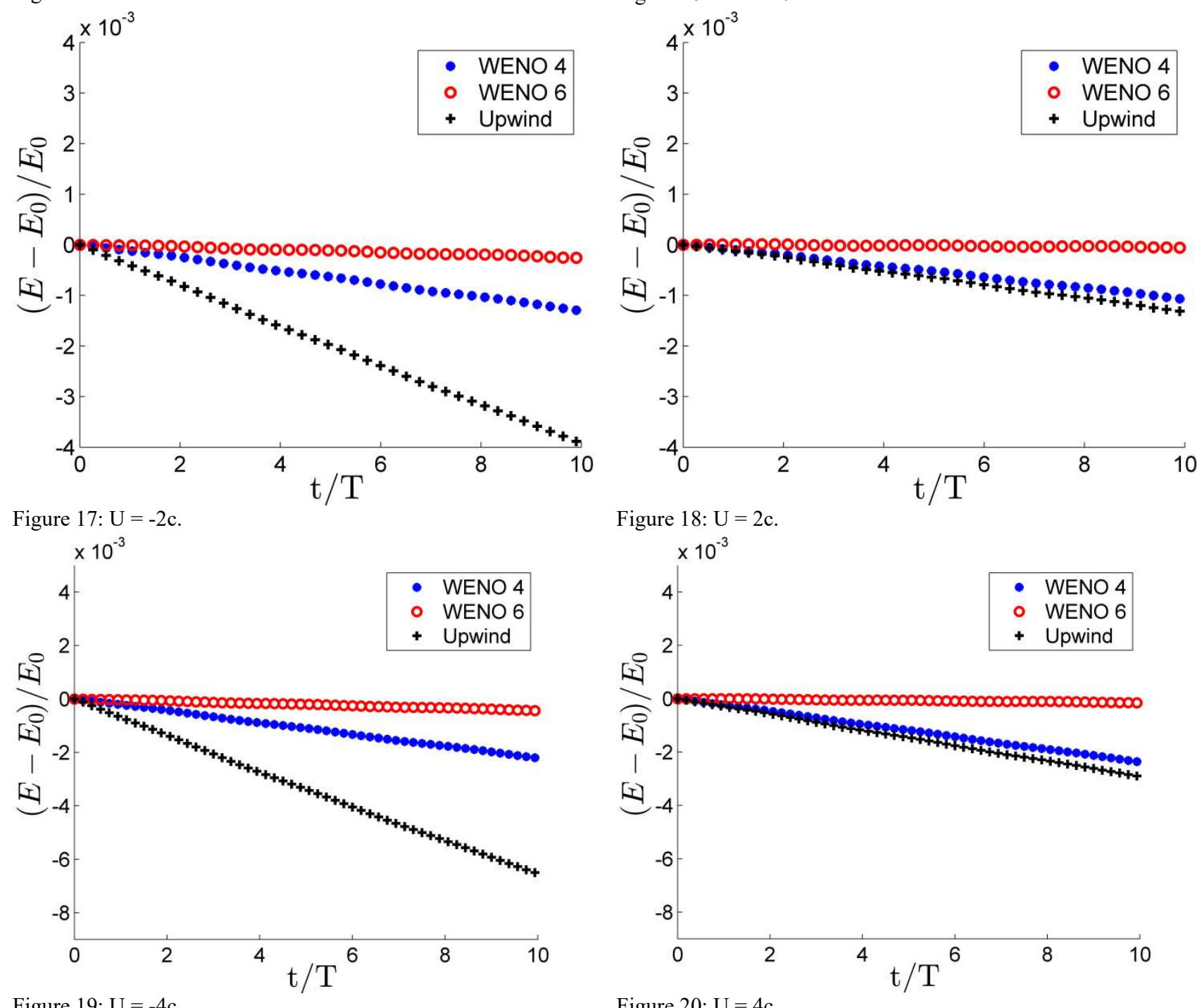

Figure 18: $U=2 c$.

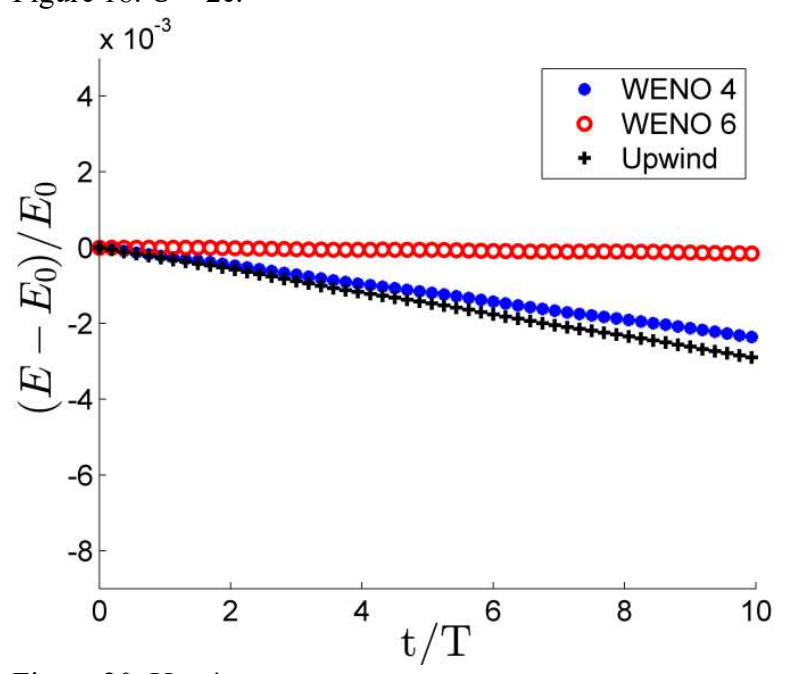

Figure 20: $U=4 c$.

Figure 19: $U=-4 c$. 


\section{CONCLUSION}

A new way of deriving finite-difference WENO coefficients has been presented together with a new smoothness indicator that adds flexibility to the schemes and is straight-forward to implement numerically without the need for tabulated coefficients. The method has been tested on the non-linear wave propagation problem in a moving frame of reference which mimics a forward speed seakeeping problem. The proposed high-order WENO scheme remains upwinded and stable under all combinations of wave speed and frame of reference speed. A massively parallel implementation of the method in 3D in the library of Glimberg et al. [14] and testing with ship geometries is in progress and will be reported on in the near future.

\section{REFERENCES}

[1] Engsig-Karup AP, Bingham HB, Lindberg O. An efficient flexible-order model for 3d nonlinear water waves [J]. JCP, 2009, 228, 2100-2118.

[2] Engsig-Karup AP, Madsen MG, Glimberg SL. A massively parallel gpu-accelerated model for analysis of fully nonlinear free surface waves [J]. Int. J. Numer. Meth. Fluids, 2012, 70(1), 20-36.

[3] Lindberg O, Bingham HB , Engsig-Karup AP. Towards Real Time Simulation of Ship-Ship InteractionPart III: Immersed Body Boundary Condition and Double Body Ship-Ship Interaction [C]. IWWWFB 29, 2014.

[4] Afshar MA, Bingham HB, Read R. A high-order finite-difference solver for the linearised potential flow wave resistance problem on curvilinear overset grids [C]. IWWWFB 29, 2014.

[5] Bingham HB, Afshar MA, Read R, Engsig-Karup AP. Stable finite difference discretizations of the forward speed seakeeping problem [C]. IWWWFB 29, 2014.

[6] Liu XD, Osher S, Chan T. Weighted ENO Schemes for Hamilton-Jacobi Equations [J]. JCP, 1994, 115, 200-212.

[7] Yamaleev NK, Carpenter MH. A systematic methodology for constructing high-order energy stable WENO schemes [J]. JCP, 2009, 228, 4248-4272.

[8] Jiang G-S, Shu C-W. Efficient implementations of Weighted ENO schemes [J]. JCP, 1996, 126, 202-228.

[9] Balsara DS, Shu C-W. Monotonicity preserving weighted essentially non-oscillatory schemes with increasingly high order of accuracy [J]. JCP, 2000, 160, 405-452.

[10] Borges R, Carmona M, Costa B, Wai Sun Don. An improved weighted essentially non-oscillatory scheme for hyperbolic conservation laws [J]. JCP, 2008, 227, 3191-3211.

[11] Bingham HB, Zhang H. On the accuracy of finite difference solutions for nonlinear water waves [J]. J ENG MATH, 2007, 58, 211-228.

[12] Osher S, Fedkiw R. Level set methods and dynamic implicit surfaces [M]. Springer-Verlag, 2002, New York.

[13] Crandall MG, Lions PL. Two approximations of solutions of Hamilton-Jacobi equations. Math. Comp. [J], 1984, 43, 1-19.

[14] Glimberg SL, Engsig-Karup AP, Nielsen AS, Dammann B. Development of software components for heterogeneous many-core architectures. Designing Scientific Applications on GPUs [M], Chapman and Hall/CRC, 2013, 73-104. 